\section{A source of highly spin-polarised slow electrons based on the 'Fano effect' on caesium atoms}

\author{
R Möllenkamp † and U Heinzmann $\ddagger$ \\ † Physikalisches Institut der Universität Münster, Domagkstr., \\ D 4400 Münster, Federal Republic of Germany \\ \$ Fritz-Haber-Institut der Max-Planck-Gesellschaft, \\ Faradayweg 4-6, D 1000 Berlin 33, Federal Republic of \\ Germany
}

\section{Received 26 October 1981}

Abstract. The source of spin-polarised electrons recently used in a 'triple-scattering experiment' in Münster is described in detail. The polarised electrons are generated by the photoionisation of caesium atoms with circularly polarised uV radiation from a high-pressure mercury discharge lamp (Fano effect). Electron intensities of $5 \mathrm{nA}$ with polarisation of $84 \%$ have been routinely obtained during continuous runs of up to 200 hours in the kinetic energy range between 17 and $500 \mathrm{eV}$ with an energy spread of $\leqslant 1.5 \mathrm{eV}$. The polarisation and intensity fluctuation was $\pm 1 \%$ and $\pm 10 \%$ in 10 hours, respectively.

\section{Introduction}

One of the novelties in atomic physics is the study of the change of electron spin polarisation due to elastic scattering of polarised electrons from unpolarised atoms. As reviewed by Kessler (1976), detailed information about the scattering amplitude $f$, the spin-flip amplitude $g$ and the relative phase between these two processes can be obtained by this type of experiment. Indeed, a recent experiment with mercury atoms (Berger et al 1981) has shown this experimental determination of amplitudes and relative phases in elastic electron scattering.

One of the important preconditions for the successful performance of this difficult experiment was the development of a suitable source of polarised electrons. The answer to the question of which type of source is the best cannot be given in general but depends in fact upon the special requirements of the experiment to be performed. The requirements of the Münster experiment for the source were firstly a high degree of polarisation because the change of polarisation itself had to be studied, secondly a small energy spread of the electrons in a wide range of kinetic energy, and thirdly a long-term stability of polarisation and intensity because the measurement of one experimental point took a couple of hours. Furthermore the ease of polarisation-reversal to eliminate apparatus-related sources of errors in the spin-dependent scattering process as well as the easy handling of the source in high vacuum not far away from the mercury atomic beam were important conditions.

The purpose of this paper is neither to give a review of the existing sources for polarised electrons nor to compare one type of source with another one nor to review the applications of polarised electron beams in general. This has been carefully done in the past by a lot of review articles (Raith 1969, Jost and Zeman 1971, Jost 1972, Kessler 1973, 1976, Lubell 1977, Celotta and Pierce 1980). The only purpose of the present paper is to describe the properties of the source used for the "triple scattering experiment' mentioned above and performed recently by Berger et al (1981).

The experimental setup for producing the polarised electrons (Fano effect) is similar to a combination of those described by Heinzmann et al (1970) and Wainwright et al (1978) taking into account special modifications to increase the intensity and polarisation of the electron beam and to improve the kinetic energy spread and other electron optical behaviour. This will be discussed in the following sections.

\section{The physical method of the Fano source}

As predicted by Fano (1969) photoelectrons emitted by alkali atoms which are exposed to circularly polarised uv radiation are highly polarised. The production of the polarised electrons can be understood to be a spin-polarisation transfer from the spinpolarised photons absorbed on to the electrons produced. The mechanism of this transfer is caused by the spin-orbit interaction because without the existence of the spin-orbit interaction the photon spin would reappear as orbital angular momentum and not as the spin of the photoelectrons.

Therefore, in order to get polarised electrons the influence of the spin-orbit interaction has to be resolved by performing the experiment at different photon energies. This can easily be seen in the spin-polarisation results (Heinzmann et al 1970) of photoelectrons ejected by caesium atoms shown in the lower part of figure 1. The polarisation has the opposite sign in different wavelength regions. On the assumption that the photoionisation cross section as well as the spectral intensity of the radiation would be constant over all wavelengths, an integration of the polarisation curve over all wavelengths would yield an unpolarised beam because the values of opposite sign in different energy regions would cancel one another. Therefore it is not surprising in atomic and molecular physics that the existence of polarised photoelectrons has been experimentally

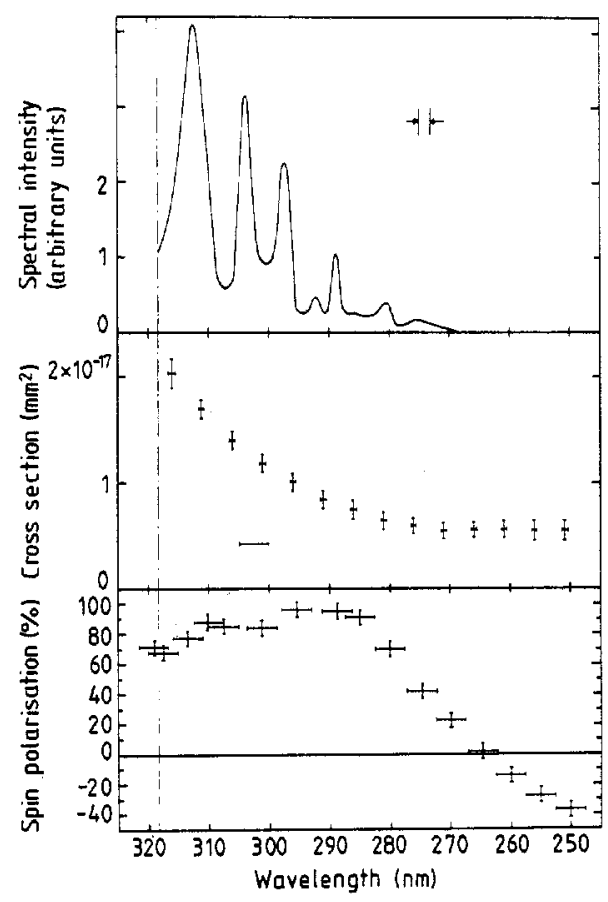

Figure 1. Fano effect in caesium atoms (Heinzmann et al 1970). Upper part: spectral intensity of the high-pressure $\mathrm{Hg}$ discharge lamp HBO 500 (Osram); middle part: photoionisation cross section; lower part: measured electron polarisation. 
proved to be a common rule rather than an exception if the influence of the spin-orbit interaction in the photoionisation is resolved via the photons absorbed, or the photoelectrons emitted (for a review see Kessler 1976, Heinzmann 1980).

To use the Fano effect as the basis of a source for polarised electrons the atoms have to be photoionised at one wavelength where the corresponding electron polarisation is very high (as performed by Drachenfels et al (1975), using rubidium atoms and laser radiation) or in a wide wavelength range using the undispersed spectrum of a powerful uV lamp (as discussed by Wainwright et al (1978) and in this paper). But the second method is only successful if the spectrum of the UV radiation absorbed is complete within the wavelength range of high spinpolarisation values (and of only one sign).

Figure 1 shows that the spectrum of the mercury highpressure discharge lamp (Osram HBO 500) fulfils this precondition in connection with the corresponding photoionisation cross section (figure 1 middle part) and the electron polarisation (figure 1 lower part) for caesium atoms. It is worthwhile to mention that Wainwright et al (1978) have used another type of UV lamp (Xe-Hg discharge lamp Hanovia) for intensity reasons but with the disadvantage that it also produced a lot of UV light between 250 and $280 \mathrm{~nm}$ (where the electron polarisation changes its sign) resulting in an average electron polarisation which is a factor of 1.33 lower than obtained in the present work.

\section{Experimental setup}

The experimental arrangement of the Fano effect source for polarised electrons consisted of three main parts:

(i) source of circularly polarised uv radiation;

(ii) oven system for the Cs atomic beam;

(iii) photoelectron extraction.

The whole source except for the uv optical components was built in a stainless steel vacuum vessel (diameter $250 \mathrm{~mm}$ and length $400 \mathrm{~mm}$ ) sealed by viton 0 -rings and pumped by a standard diffusion pump $\left(400 \mathrm{l} \mathrm{s}^{-1}\right)$ to a vacuum of about $10^{-1} \mathrm{mPa}$ (with the atomic beam on), because high vacuum was

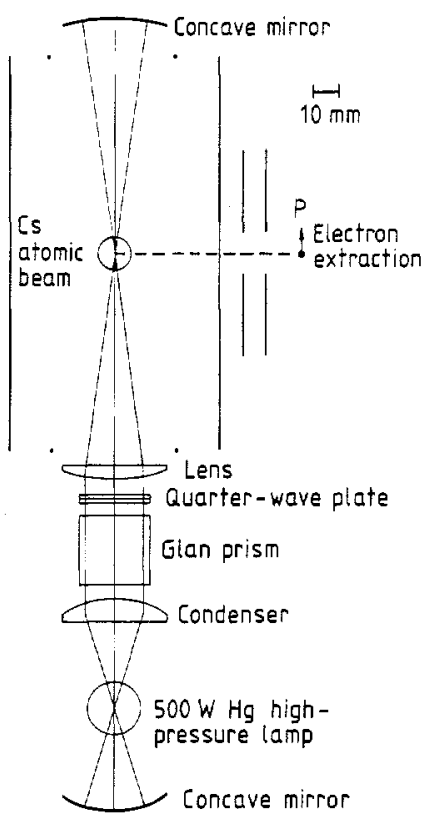

Figure 2. Schematic horizontal cross section of the UV source and of the electron extraction. sufficient for this type of source. The extraction system is described in $\S 4.3$.

\subsection{Uv-radiation source}

Figure 2 shows a schematic diagram of the uv optical components and of the electron extraction used. The UV radiation was produced by means of a mercury high-pressure discharge lamp HBO $500 \mathrm{~W}-2$ (Osram). It had an electrical power of $500 \mathrm{~W}$, an average photon flux density of $300 \mathrm{~cd} \mathrm{~cm}^{-2}$ and a discharge size of $4 \mathrm{~mm} \times 1 \mathrm{~mm}$. The intensity of the UV light in the wavelength range of interest was about $15 \%$ of the total UV and visible radiation intensity emitted. It is worthwhile to mention that we did not use the HBO $1000 \mathrm{~W}$ mercury discharge lamp analogous to the $1000 \mathrm{~W} \mathrm{Hg}-\mathrm{Xe}$ radiation source used by Wainwright et al (1978), although the $1000 \mathrm{~W}$ source emits twice the intensity. However, this is only due to the fact that the size of the discharge is twice that of the $500 \mathrm{~W}$ type. As the size of the light source is nearly proportional to the energy spread of the photoelectrons produced, as shown in $\S 4.3$, the doubling of the photon flux using a $1000 \mathrm{~W}$ lamp instead of a $500 \mathrm{~W}$ one would directly lead to more than doubling of the kinetic energy spread of the polarised electrons.

The solid angle of the radiation emitted by the lamp and accepted by the condenser as well as by the concave mirror was about 1 sr. All lenses and mirrors used and shown in figure 2 were made of the silica-glass Herasil. Both mirrors were coated by $\mathrm{Al}+\mathrm{MgF}_{2}$ in order to get a higher reflectivity. The condenser (Halle, Berlin) consisted of three lenses and had a focal length of $50 \mathrm{~mm}$ and a diameter of $40 \mathrm{~mm}$. The condenser and the concave mirror (radius $50 \mathrm{~mm}$ ) were placed $50 \mathrm{~mm}$ away from the mercury lamp. The radiation was linearly polarised by a Glan air prism (cross section $35 \mathrm{~mm} \times 35 \mathrm{~mm}$, length $35 \mathrm{~mm}$, made by Steeg and Reuter, Frankfurt). The maximum divergence of the radiation in the Glan prism was $\pm 2.5^{\circ}$ which was smaller than the maximum angle of acceptance $\left( \pm 4^{\circ}\right)$ of the prism. Therefore it completely reflected out radiation of the wrong polarisation state. This light reflected at the air layer in the prism left it through a window at the side. The radiation was circularly polarised by a quartz quarter-wave double plate rotated by $45^{\circ}$ with respect to the linear polariser; two plates (cross section $35 \mathrm{~mm} \times 35 \mathrm{~mm}$ ) had an angular difference of $90^{\circ}$ between their optic axes and a thickness difference resulting in an optical path difference for the two linear polarisation states of $\lambda / 4$ at $\lambda=300 \mathrm{~nm}$. The circularly polarised radiation was focused by a plane convex lens onto the $C$ s atomic beam inside the vacuum system. A second concave mirror was used to increase the intensity of the UV radiation; as the radiation was reflected only under normal incidence, depolarisation effects were not observed in the experiment. All optical components were mounted on an optical bench (Spindler + Hoyer) outside the vacuum. The circularly polarised beam had to go through two Herasil windows which had been proved to be free of any. birefringence even under atmospheric pressure.

This optical UV source whose total price was about $15000 \mathrm{DM}$ delivered about $0.2 \mathrm{~W}$ circularly polarised uv radiation in the wavelength range $280-318 \mathrm{~nm}$ and in a cross section of about $10 \mathrm{~mm} \times 3 \mathrm{~mm}$ at the photoionisation target. The photon flux there was about $10^{18}$ photons $\mathrm{s} \mathrm{cm}^{2}$ in the wavelength range of interest.

\subsection{Oven system for the Cs atomic beam}

Figure 3(a) shows a schematic diagram of this arrangement, figure $3(b)$ the corresponding photograph. The oven holds four $5 \mathrm{~g}$ glass ampoules of $\mathrm{Cs}$ metal, which could be broken under vacuum conditions. The oven had its operating temperature of about $530 \mathrm{~K}$. The caesium vapour passed a heated pipe, an UHV metal-sealed stainless steel valve for switching off the atomic beam and a glass capillary array $(50 \mu \mathrm{m}$ tube diameter, Galileo, 

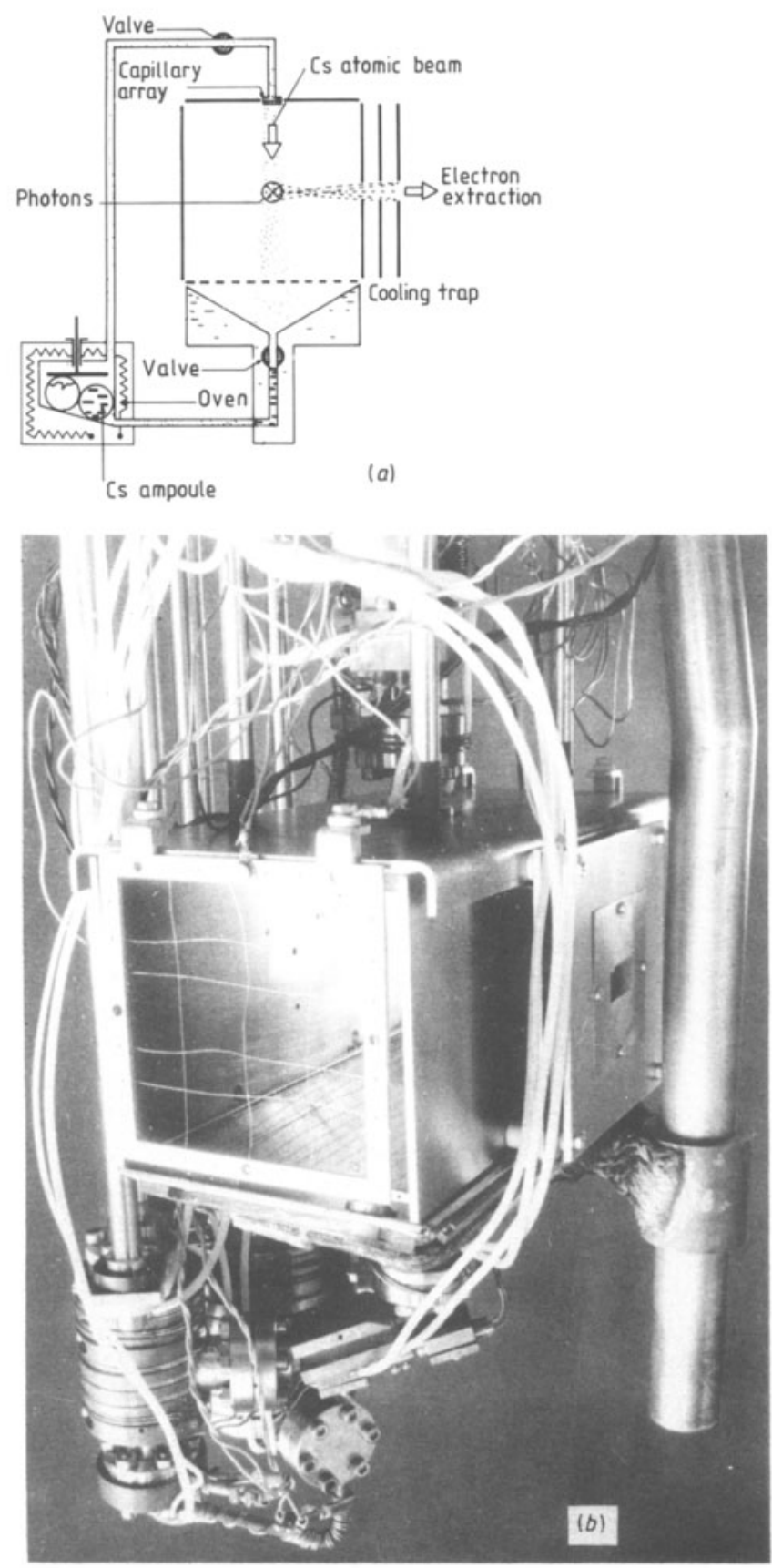

Figure 3. (a) Schematic vertical cross sections of the oven and the atomic vapour beam system; $(b)$ photograph of oven and extraction system.

Sturbridge USA) which had a temperature about $50 \mathrm{~K}$ higher than the other oven system to break alkali dimers in the beam. All hot oven parts were heated resistively by different bifilary coiled heating-wires (Philips). The atomic beam was frozen by a liquid- $\mathrm{N}_{2}$-cooled trap. When, after 120 hours, the oven was empty, the source was stopped for 2 hours to increase the temperature in the cooling trap to $320 \mathrm{~K}$ so that the caesium metal could flow back as liquid through the open valve to the oven. Then the atomic beam cycle started again for a further 80 hours. The main advantage of this cycle was the long duration Cs beam which could also be interrupted by periods when the apparatus was under atmospheric pressure. In this case both valves shown in figure 3 were closed in order to save the Cs content (500 DM).

\section{Results}

\subsection{Method of polarisation reversal}

In the experimental studies of the Fano effect in caesium (Heinzmann et al 1970) and thus also in the work described here, all photoelectrons produced were extracted by an electric field regardless of their direction of emission. For reasons of symmetry the direction of the spin-polarisation vector was defined by the photon spin for this experimental arrangement. The spin polarisation of the photoelectrons produced was proportional to the spin polarisation (i.e. degree of circular polarisation) of the photons absorbed. Therefore the easiest way to reverse the electron polarisation was the reversal of the optical polarisation rotating the quarter-wave plate by $90^{\circ}$. This procedure had the main advantage that intensity, polarisation (except for its sign) and position of the photon beam and therefore all figures of merit of the polarised electron beam were stable during the sign change of the polarisations.

Figure 4 shows the signal difference of the two ratemeters connected with the counting channels in the Mott detector as a

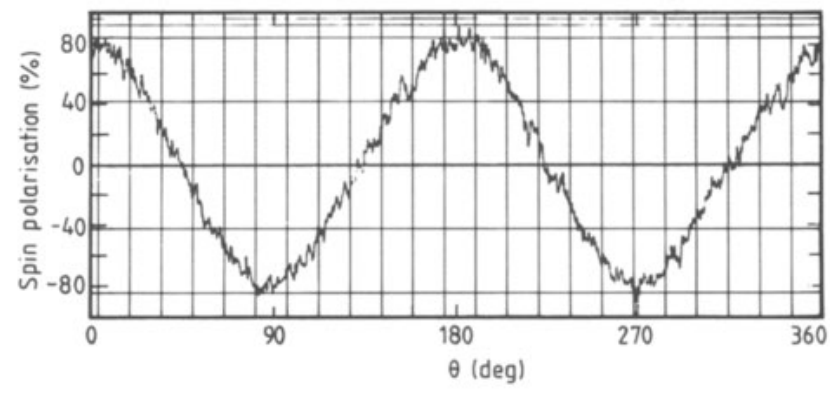

Figure 4. Electron polarisation measured as function of the quarter-wave plate orientation.

function of the rotation angle between prism and quarter-wave plate. Because this count rate difference is given by $\Delta N=$ $\bar{N} \times P \times S$ (Kessler 1976) where $P$ is the electron polarisation and $S$ the asymmetry function of the Mott detector and $\bar{N}$ is the sum of the two count rates, the ordinate in figure 4 can be scaled in units of the electron polarisation assuming that the primary beam intensity was constant with time. The Sherman function $S$ has been obtained by an experimental method similar to that described by Jost and Kessler (1966) and Heinzmann (1978). Figure 4 shows that the electron polarisation follows directly the degree of circular polarisation of the UV light. As pointed out by Heinzmann (1977) the circular polarisation is given by $P_{\text {circ }} \propto 2 A B \times\left(A^{2}+B^{2}\right)^{-1}$ and the amplitudes of the two linear oscillations $A$ and $B$ forming the polarisation ellipse are given by $E_{0} \times \sin \theta$ and $E_{0} \times \cos \theta$, respectively. Therefore the curve in figure 4 has the shape of $\sin \theta \times \cos \theta$ shifted by $45^{\circ}$ which is the angle between the axes of prism and quarter-wave plate mentioned in $\$ 3.1$.

The source yielded polarisation values of more than $80 \%$ at electron currents up to $10 \mathrm{nA}$. The fact that the sign of the electron polarisation could be easily and even quickly changed was a necessity for the 'triple-scattering experiment' of Berger $\boldsymbol{e t}$ al (1981) in order to eliminate apparatus-related asymmetries of the experimental results in the electron scattering.

\subsection{Stability}

For this type of experiment it was indispensable to have stable conditions of the source over many hours, as demonstrated in figure 5 where the electron polarisations measured are shown as 


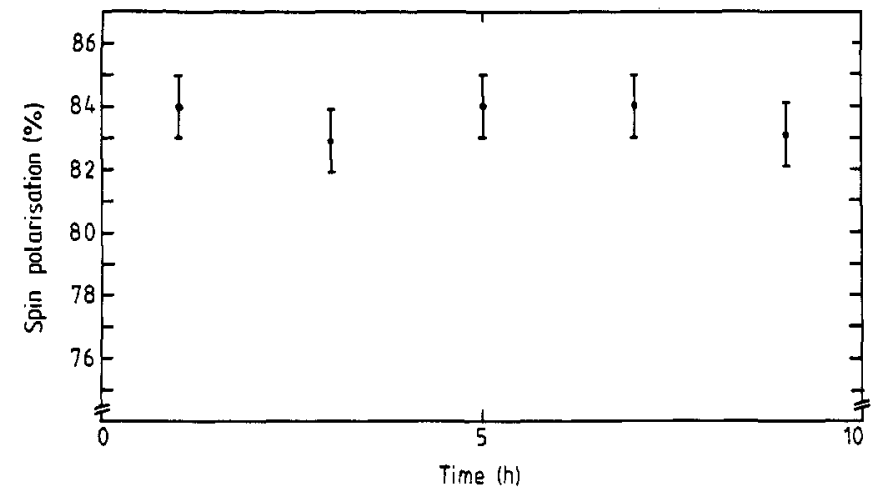

Figure 5. Electron polarisation measured as a function of the time.

a function of the time. The error bars are the single statistical errors of the polarisation analysis not taking into account the uncertainty of the Sherman function $S$ of the Mott detector which was of course time independent. Figure 5 shows that the polarisation fluctuation $\triangle P / P$ was smaller than $\pm 1 \%$ in 10 hours.

\subsection{Electron optical behaviour of the polarised electron beam} Beside the intensity and the polarisation the electron beam had to fulfil other preconditions to be used as a source in the experiment mentioned: a wide kinetic energy range of the electrons, a small energy spread and a high brightness of the beam.

The main part of the electron extraction system was a quadrupole field which ensured that all photoelectrons produced resulting from the photoionisation of the atomic vapour beam (and only these) were collected. The quadrupole field shown in figure 6 is the same as that described by Heinzmann (1978) except that the electric potentials were reduced by a factor of 10 . As shown in figure 3 the lower plate was in practice a system of parallel thin wires (distance $5 \mathrm{~mm}$ ) to transmit the $\mathrm{Cs}$ atomic beam coming from the top. Using this arrangement no radiation could reach any of the plates. The equipotential lines (full curves

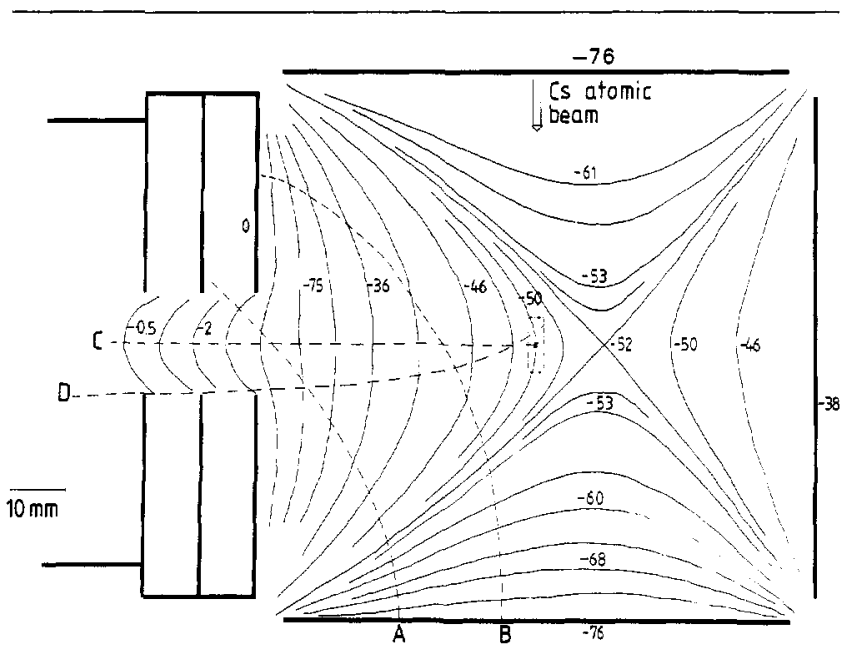

Figure 6. Vertical cross section of the extraction system with the equipotential lines (full curves, numbers are the potential) and the electron paths (broken curves). The cross section of the radiation beam is indicated by the chained rectangle. in figure 6) have been obtained by a measurement using a twodimensional model drawn on conductivity paper. These and the paths of the electrons (broken curves) have been confirmed by computer calculations.

The potential of the upper and lower plate was negative compared with the position where the photoelectrons were produced. The potentials of the plates on the left and right were positive with respect to the target, but the quadrupole was not symmetric because of the different potentials with a saddle point lying on the right-hand side of the target. All photoelectrons produced were extracted from the left-hand side on to the apertures (curves $C$ and $D$ in figure 6), because they had a kinetic energy smaller than $1 \mathrm{eV}$, which was not sufficient to overcome the saddle point. But electrons which might come from the right electrode must have more than $14 \mathrm{eV}$ kinetic energy in order to surmount the saddle point. Electrons which were produced at the upper or lower plate could pass the quadrupole field, but they had a divergence which was too large to be accepted by the second stage of the extraction system (figure 6, curves $A$ and $B$ ). There the electron beam had a kinetic energy of $50 \mathrm{eV}$ and an energy spread of $\leqslant 1.5 \mathrm{eV}$ (FWHM) (given by the bandwidth of the radiation used $(0.5 \mathrm{~V}$, see $\S 2)$ and by the field of $0.3 \mathrm{~V} \mathrm{~mm}^{-1}$ in the target together with the target size) which has been measured by means of a filter lens.

Additional thin wires with a negative potential (not shown in figure 6 , but in figure 2 as points in cross section) enabled the quadrupole to focus the electron beam in the direction of light perpendicular to the plane shown in figure 6 , too. In the quadrupole the magnetic fields were compensated to be better than $5 \mathrm{mOe}$, using two pairs of Helmholtz coils.

The $50 \mathrm{eV}$ electrons in the second stage of the extraction system could be accelerated or decelerated in a further electron lens in order to be focused onto the mercury atomic beam with different kinetic energies.

The photoelectrons extracted perpendicularly to the light beam were transversely polarised. Figure 7 shows the spinpolarisation values measured with the Mott detector $(120 \mathrm{kV}$ ) as a function of the kinetic energy of the electrons (at the position of the mercury atomic beam). An energy range from 17 to $500 \mathrm{eV}$ was achieved in the experiment. As also shown in figure 7 the electron polarisation has been found to be nearly independent from the electron energy. It is worthwhile to point out that the polarisation was not found to degrade substantially due to low-energy electron caesium spin exchange collisions as supposed by Celotta and Pierce (1980) if small extraction fields were used. Even at a field of $0.3 \mathrm{~V} \mathrm{~mm}^{-1}$ or at kinetic energies of only a few $\mathrm{eV}$ this depolarisation effect did not happen. As figure 7 shows, the polarisations measured were higher than

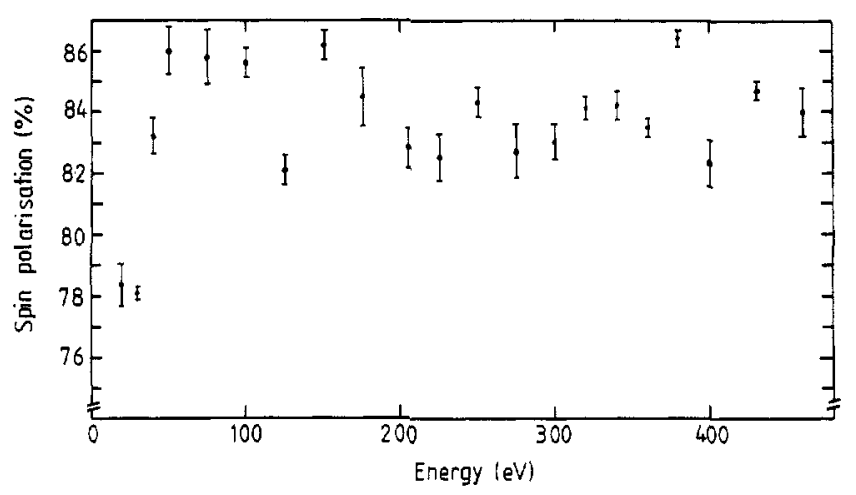

Figure 7. Electron polarisation measured as function of the kinetic energy in the $\mathrm{Hg}$-scattering stage. 
Table 1. Figures of merit for Fano-type sources.

\begin{tabular}{lcc}
\hline & This work & Wainwright et al \\
\hline Current $I_{\text {av }}$ routinely obtained & $5 \mathrm{nA}$ & $10 \mathrm{nA}$ \\
Current $I_{\max }$ & $10 \mathrm{nA}$ & $25 \mathrm{nA}$ \\
Electron polarisation $P$ & 0.84 & 0.63 \\
$P^{2} \cdot I_{\text {av }}$ & $4 \mathrm{nA}$ & $4 \mathrm{nA}$ \\
Output energy & $\geqslant 17 \mathrm{eV}$ & $1000 \mathrm{eV}$ \\
Energy spread & $\leqslant 1.5 \mathrm{eV}$ & $3 \mathrm{eV}$ \\
Invariant brightness $b_{\text {inv }}$ & $0.04 \mathrm{nA} \mathrm{mm} \mathrm{mr}^{-1} \mathrm{eV}^{-1}$ & $0.02 \mathrm{nA} \mathrm{mm} \mathrm{mr}^{-1} \mathrm{eV}^{-1}$ \\
Emittance $\varepsilon$ at $1 \mathrm{keV}$ & $100 \mathrm{mrad} \mathrm{mm}$ & $200 \mathrm{mrad} \mathrm{mm}^{-1}$ \\
Lifetime & $200 \mathrm{~h}$ & $75 \mathrm{~h}$ \\
Stability $\Delta P / P$ & $\pm 1 \% / 10 \mathrm{~h}$ & \\
Stability $\Delta I_{\text {av }} / I_{\text {av }}$ & $\pm 10 \% / 10 \mathrm{~h}$ & optical \\
Polarisation reversal & optical & \\
\hline
\end{tabular}

$82 \%$ (except for the two values of $78 \%$ where the electrons have been strongly decelerated in the third part of the extraction).

Another point of interest was to achieve a collimated beam. The brightness $b$ is the current density emitted into the unit solid angle. The more parallel the beam and the higher its current density, the higher its brightness $b$ is. But it has to be taken into account, however, that the angular spread of an electron beam decreases when the electrons are accelerated (see for example Jost 1972). In order to have an energy-independent figure of merit the 'invariant brightness' $b_{\text {inv }}=b \times E^{-1}=I \times \pi^{-2} \times \varepsilon^{-2} \times$ $E^{-1}$ is introduced where $E$ is the kinetic energy, $b$ the brightness, $I$ the current and $\varepsilon$ the emittance of the electron beam. In our case $b_{\text {inv }}$ has been measured to be $0.04 \mathrm{nA} \mathrm{mm}^{-2} \mathrm{sr}^{-1} \mathrm{eV}^{-1}$.

\section{Summary of figures of merit and conclusion}

In order to have an overview of all figures of merit of the source discussed, table 1 shows the data in comparison with the corresponding results by Wainwright et al (1978). The comparison shows very similar data especially with respect to the electron optical behaviour of the beam $\left(P^{2} \times I\right.$, brightness, emittance). The advantages of the source discussed in the present paper were the high spin polarisation of $84 \%$, the wide energy range from 17 to $500 \mathrm{eV}$ and the small energy spread of $\leqslant 1.5 \mathrm{eV}$. All these features were necessary preconditions of successfully performing the 'triple-scattering experiment' recently done in Münster. It is worthwhile mentioning that this Fano-type source has been developed and used for this experiment even though a GaAs-type source (Celotta and Pierce 1980) has been built up at the same time in the same lab. Further advantages of the source described in this paper were the very easy handling of the system in high vacuum, the longterm stability of all components and last but not least the comparatively low cost of the apparatus.

\section{Acknowledgments}

The authors want to express their gratitude to Professor $\mathrm{J}$ Kessler for his continued interest and encouragement. We thank Dr K Kollath, Dr W Wübker and O Berger for their assistance in testing the source described, and acknowledge gratefully $\mathrm{Dr} \mathrm{K}$ Jost for reading the manuscript and making valuable suggestions.

\section{References}

Berger O, Kessler J, Kollath K J, Möllenkamp R and Wübker W 1981 'Triple' scattering experiment for obtaining the maximum possible information on elastic electron scattering from mercury

Phys. Rev. Lett. 46 768-70
Celotta R J and Pierce D T 1980 Sources of polarized electrons Adv. At. Mol. Phys. 16 101-57 (New York: Academic)

Drachenfels W v, Koch U T, Müller Th M and Schaefer H R 1975 Direct measurement of Fano-effect on $\mathrm{Rb}$ at $266 \mathrm{~nm}$ Phys. Lett. 51A 445-6

Fano U 1969 Spin orientation of photoelectrons ejected by circularly polarized light

Phys. Rev. 178 131-6

Heinzmann U 1977 An apparatus for the production of circularly polarised vuv radiation

J. Phys. E: Sci. Instrum. 10 1001-5

Heinzmann U 1978 New vacuum-ultraviolet absorption data for lead vapour obtained by spin-polarisation measurements J. Phys. B: At. Mol. 11 399-412

Heinzmann U 1980 Spin polarized photoelectrons from atoms and molecules

Appl. Opt. 19 4087-91

Heinzmann U, Kessler J and Lorenz J 1970 ElektronenSpinpolarisation bei der Photoionisation unpolarisierter Cäsiumatome mit zirkular polarisiertem Licht

Z. Phys. 240 42-61.

Jost K 1972 Spin polarized electron beams Proc. VI Yugoslav Symp. and Summer School on the Physics of Ionized Gases published by the Inst. of Phys. Beograd (ed. V Kurepa) pp 37-64

Jost K and Kessler J 1966 Zur Polarisation langsamer Elektronen durch Streuung an Quecksilber zwischen 180 und $1700 \mathrm{eV}$

Z. Phys. 195 1-12

Jost K and Zeman H D 1971 Production of polarized electron beams

HEPL Report 590 Stanford (unpublished)

Kessler J 1973 Polarized electron sources

At. Phys. Vol. 3 (New York: Plenum) pp 523-41

Kessler J 1976 Polarized Electrons (Berlin: Springer)

Lubell M S 1977 Polarized electrons

At. Phys. Vol. 5 (New York: Plenum) pp 325-73

Raith W 1969 Polarized electrons

At. Phys. Vol. 1 (New York: Plenum) pp 389-415

Wainwright P F, Alguard M J, Baum G and Lubell M S 1978

Application of a dc Fano effect polarized electron source to low-energy electron-atom scattering

Rev. Sci. Instrum. 49 571-85 